\title{
Combined effects of grain size, flow volume and channel width on geophysical flow mobility: three-dimensional discrete element modeling of dry and dense flows of angular rock fragments
}

\author{
Bruno Cagnoli ${ }^{1}$ and Antonio Piersanti ${ }^{2}$ \\ ${ }^{1}$ Istituto Nazionale di Geofisica e Vulcanologia, Via Donato Creti 12, 40128 Bologna, Italy \\ ${ }^{2}$ Istituto Nazionale di Geofisica e Vulcanologia, Via di Vigna Murata 605, 00143 Rome, Italy \\ Correspondence to: Bruno Cagnoli (bruno.cagnoli@ingv.it)
}

Received: 20 May 2016 - Discussion started: 8 June 2016

Revised: 23 January 2017 - Accepted: 23 January 2017 - Published: 16 February 2017

\begin{abstract}
We have carried out new three-dimensional numerical simulations by using a discrete element method (DEM) to study the mobility of dry granular flows of angular rock fragments. These simulations are relevant for geophysical flows such as rock avalanches and pyroclastic flows. The model is validated by previous laboratory experiments. We confirm that (1) the finer the grain size, the larger the mobility of the center of mass of granular flows; (2) the smaller the flow volume, the larger the mobility of the center of mass of granular flows and (3) the wider the channel, the larger the mobility of the center of mass of granular flows. The grain size effect is due to the fact that finer grain size flows dissipate intrinsically less energy. This volume effect is the opposite of that experienced by the flow fronts. The original contribution of this paper consists of providing a comparison of the mobility of granular flows in six channels with a different cross section each. This results in a new scaling parameter $\chi$ that has the product of grain size and the cubic root of flow volume as the numerator and the product of channel width and flow length as the denominator. The linear correlation between the reciprocal of mobility and parameter $\chi$ is statistically highly significant. Parameter $\chi$ confirms that the mobility of the center of mass of granular flows is an increasing function of the ratio of the number of fragments per unit of flow mass to the total number of fragments in the flow. These are two characteristic numbers of particles whose effect on mobility is scale invariant.
\end{abstract}

\section{Introduction}

The prediction of mobility of geophysical flows is one of the most important research goals in the earth sciences. The geophysical flows we are interested in here include rock avalanches (Hungr et al., 2014) and the underflow of pyroclastic flows such as block-and-ash flows (Cas and Wright, 1988). These flows of angular rock fragments are dense and dry and they can be considered as some of the most hazardous natural phenomena. Rock avalanches are dry because their extensive fragmentation during motion generates new intergranular spaces that cannot be filled by water during their relatively short travel times (Hungr et al., 2014). Blockand-ash flows are dry because they are due to the collapse of volcanic domes (Nairn and Self, 1978; Saucedo et al., 2002) or the explosive fragmentation of volcanic plugs (Hall et al., 2015) where domes and plugs are too hot for liquid water to be present.

Grain size, flow volume and channel width are among the main variables that affect the mobility of geophysical flows. Since the values of these quantities can vary significantly in nature, this variation can cause significantly different travel distances in natural flows. In the present paper, we illustrate the combined effect of grain size, flow volume and channel width on granular flow mobility. This result is built on our previous laboratory experiments (Cagnoli and Romano, 2010, 2012a) and numerical simulations (Cagnoli and Piersanti, 2015) where we focused on the quantities that enter the numerator of a scaling parameter that the apparent coefficient of friction $\mu_{\mathrm{A}}$ (i.e., the reciprocal of mobility) is proportional to. The main original contribution of our paper here consists 
of assessing the effect of the channel width on flow mobility. The channel width is a quantity that enters the denominator of this scaling parameter.

The new set of numerical simulations illustrated in this paper confirms that the finer the grain size (all the other features being the same), the more mobile the center of mass of the granular flow (Cagnoli and Romano, 2012a; Cagnoli and Piersanti, 2015). For example, we expect that in nature the fragmentation during motion of rock clasts with different hardness can generate flows with different grain size. There is field evidence showing that rock avalanches of harder rocks tend to be less mobile than those of weaker rocks (Zhang and Yin, 2013). Importantly, we demonstrate that finer grain size flows are intrinsically less energetically dissipative than coarser grain size flows, irrespective of the presence of an interstitial fluid (either gaseous or liquid). Our new set of simulations also confirms that the larger the flow volume (all the other features being the same), the less mobile the center of mass of the granular flows (Okura et al., 2000; Cagnoli and Romano, 2012a; Cagnoli and Piersanti, 2015). The mobility of the center of mass is different from the mobility of the flow front, which becomes more distal as flow volume increases (Scheidegger, 1973) because the larger the flow volume, the longer the longitudinal spreading of the flow and its deposit (Davies, 1982; D'Agostino et al., 2010). This is so, in a channel for example, because the planimetric area inundated by a flow and its deposit is proportional to a power of the flow volume (Griswold and Iverson, 2008).

Here we illustrate the results of three-dimensional numerical simulations carried out by means of a discrete element method (DEM) that we have shown to correctly predict the relative mobility of laboratory granular flows with different features (Cagnoli and Piersanti, 2015). It is because of this previous experimental validation of our computer modeling that we rely on its predictive power to replace labor-intensive and time-consuming laboratory experiments. This is particularly useful in this paper where we compare the mobility of flows in six different channels with a different cross section each, whose construction and use in laboratory experiments would be truly demanding. Discrete element modeling considers particle-particle and particle-boundary interactions and, for this reason, it is able to correctly estimate the relative energy dissipation of the flows without prior parameter tuning. Conversely, models of geophysical flows that ignore their granular nature (such as multiphase and depthaveraged models) are unable to predict the flow runouts even with the correct parameter values. Studies of granular flows by means of discrete element modeling include Valentino et al. (2008), Banton et al. (2009), Girolami et al. (2012), Yohannes et al. (2012), Mollon et al. (2012), Cagnoli and Piersanti (2015) and Mead and Cleary (2015).

Our new numerical simulations confirm that the narrower the channel (all the other features being the same), the smaller the mobility of the center of mass of the granular flow. Therefore, the channel width (together with the length of the flow or that of the deposit) enters the denominator of a new scaling parameter $\chi$ that gives rise to a highly significant linear correlation with the reciprocal of mobility $\mu_{\mathrm{A}}$. This parameter is new because the exponent of the channel width is shown here to be equal to 1 . Parameter $\chi$ implies that the mobility of the center of mass of granular flows is an increasing function of the ratio of the number of particles per unit of flow mass to the total number of particles in the flow.

Although there are many more variables (besides grain size, flow volume and channel width) that can affect the mobility of geophysical flows in nature (such as the initial speed, the subsurface friction, the intergranular fluid density etc.), the fact that their values are constant or equal to zero in our analysis is not a shortcoming. In fact, to be useful, a model has to single out a single phenomenon. The introduction of too much complexity can hinder the comprehension of the effect of a variable in a system where different phenomena interfere. It is also not the actual size of the examined granular flows (either in a laboratory experiment or numerical simulation) that matters. Even if our system were 10 times larger (assuming to be able to solve the consequent practical complications in the laboratory and numerical problems in the computer simulations, such as prohibitively large computer processing times), it would still be much smaller than the corresponding natural system. The attention of the reader should rather focus on whether the phenomenon that the model is focusing on is scale invariant. The grain size effect, the flow volume effect and the channel width effect that are illustrated here are scale invariant because they are a function of only characteristic numbers of particles, which, since they are dimensionless, determine the distinctive character of any granular flow at any scale either in the laboratory or in nature.

\section{Method}

\subsection{Channels and granular material in the three-dimensional numerical simulations}

In our new three-dimensional numerical simulations, we vary the width $w$ of the concave-upward channel we used in our previous laboratory experiments (Cagnoli and Romano, 2012a) and numerical simulations (Cagnoli and Piersanti, 2015). These virtual channels were generated by means of a CAD software (Rhinoceros). Both here and in our previous works, the channels consist of a straight upper ramp and a curved chute (Figs. 1 and 2). The longitudinal profile of the curved chutes is computed by using a hyperbolic sine equation:

$z=0.3-0.085 \operatorname{arcsinh}(11.765 x)$,

where the variables are in meters. This equation represents a slightly modified version of the profile of Mayon Volcano in the Philippines (Becker, 1905). The horizontal length of the curved chutes is $1.4 \mathrm{~m}$. The granular material is placed be- 


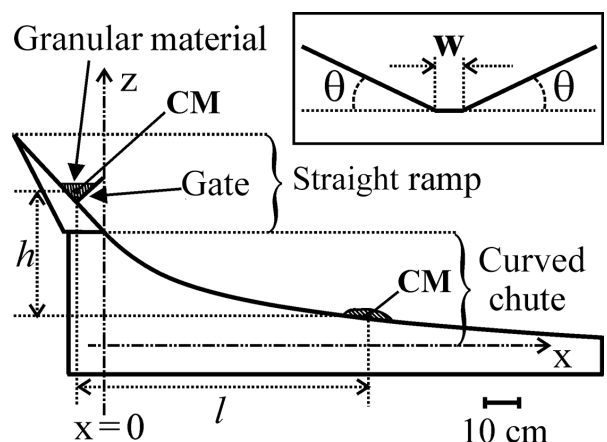

Figure 1. Longitudinal cross section of all the channels used in the numerical simulations. The inset illustrates the transversal cross section of both the straight ramps and the curved chutes where quantities $w$ (the channel width) and $\theta$ (the sidewall inclination) are shown. CM stands for center of mass.

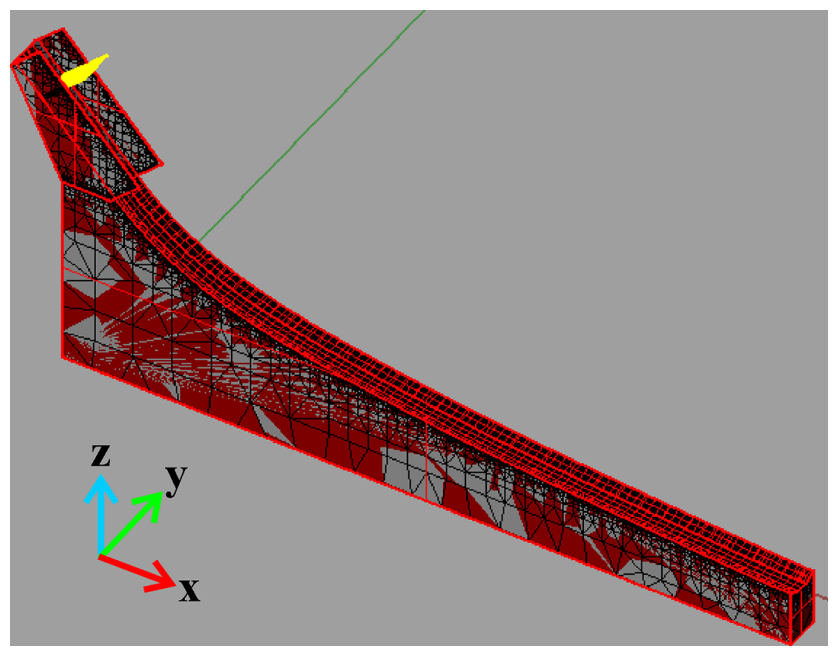

Figure 2. Example of a three-dimensional virtual channel generated by means of a CAD software (Rhinoceros) for the numerical simulations. The concave upward channel is shown in red and the gate in yellow.

hind a sliding gate located $22.3 \mathrm{~cm}$ above $x=0$, where this distance is measured along the upper ramp (Figs. 1 and 2). The gate is removed in a direction perpendicular to the surface of the upper ramp.

The upper ramps and the chutes have the same trapezoidal cross section (Fig. 1) that corresponds, in nature, to a Vshaped topographic incision with sediment infilling in the center (see, for example, the natural channel cross sections in the paper by Zhang and Yin, 2013). The width $w$ we use in the calculations is the smallest distance between the inclined sidewalls of the channels (Fig. 1). This distance has been preferred because the width at the base of a channel that is trapezoidal in cross section concerns the flows more than that at the top that is larger than the flow width. Here we adopt $w$ values equal to $6 \mathrm{~mm}$ (the same value used in our previous publications), 10, 16 and $26 \mathrm{~mm}$ (Fig. 3). Most

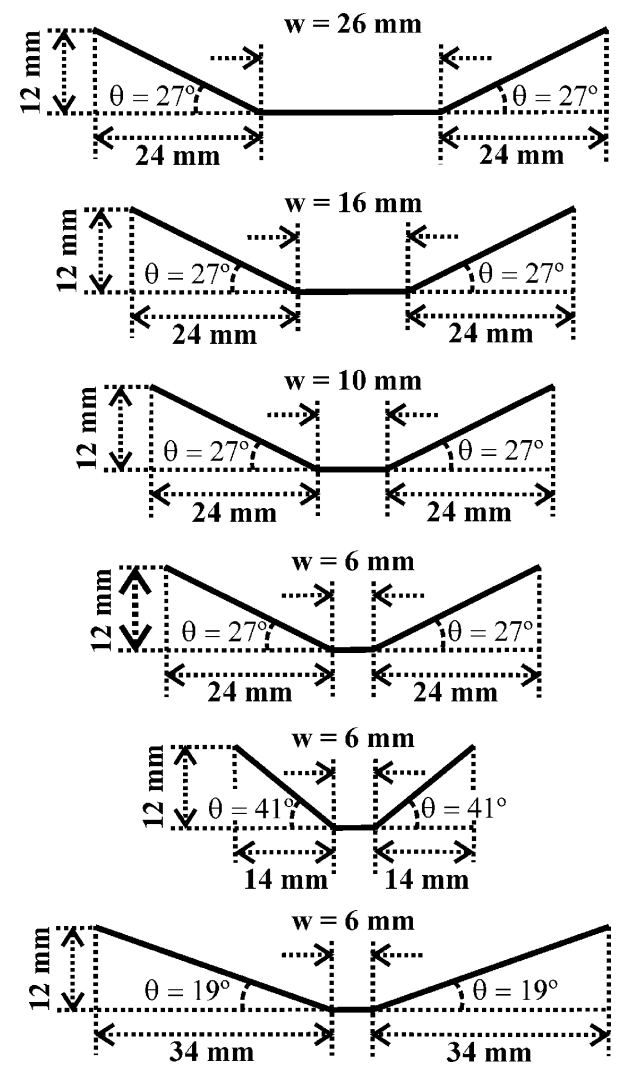

Figure 3. The different values of the channel width $w$ and the sidewall inclination $\theta$ that are adopted in the numerical simulations.

of the simulations (16) were carried out in channels with the same lateral side inclination $\theta\left(27^{\circ}\right)$, but two ancillary simulations with smaller and larger $\theta$ values ( 19 and $41^{\circ}$, respectively) were also carried out (Fig. 3).

The numerical flows are dry and consist of particles with three different shapes. We use particles with a cubic shape, half a cubic shape and a quarter of a cubic shape (Fig. 4). These polyhedrons represent an equant, an oblate and a prolate particle, respectively. Nonspherical particles are preferred because when interacting amongst themselves and with the boundary surfaces, their energy dissipation mechanism (due to collisions and attrition) is comparable with that of natural fragments since they are both angular (Cagnoli and Romano, 2012a; Mead and Cleary, 2015). The proportion of each particle shape is always the same in all flows irrespective of grain size, flow mass or channel features: the equant particles are $38 \%$, the oblate particles $22 \%$ and the prolate particles $40 \%$ of the flow mass. This generates more realistic flows than those with particles of only one shape because natural geophysical flows contain particles with different shapes. We have shown that flows with different proportions of particle shapes dissipate different amounts of energy per unit of travel distance (Cagnoli and Piersanti, 2015). For this reason, it is important that the comparison of mobility is carried out among flows with the same proportions of particle 


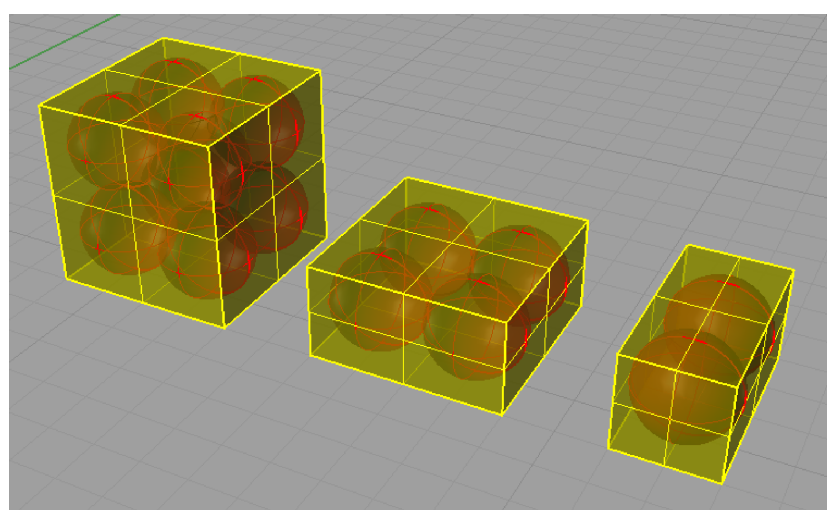

Figure 4. Shapes of the particles that are used in the numerical simulations. The inscribed clusters of spheres are also shown.

Table 1. Characteristics of channels and flows.

\begin{tabular}{lrrr}
\hline $\begin{array}{l}\text { Channel } \\
\text { width } w \\
\mathrm{~mm}\end{array}$ & $\begin{array}{r}\text { Lateral side } \\
\text { inclination } \theta\end{array}$ & $\begin{array}{r}\text { Total flow } \\
\text { mass } \\
\mathrm{g}\end{array}$ & $\begin{array}{r}\text { Grain size } \\
\delta \\
\mathrm{mm}\end{array}$ \\
\hline 6 & & 8.9 & 1 \\
6 & $27^{\circ}$ & 13 & 1 \\
6 & $27^{\circ}$ & 26 & $1,1.5,2$ \\
10 & $27^{\circ}$ & 29 & $1,1.5,2$ \\
16 & $27^{\circ}$ & 33 & $1,1.5,2$ \\
26 & $27^{\circ}$ & 39 & $1,1.5,2$ \\
26 & $27^{\circ}$ & 93 & 2 \\
16 & $27^{\circ}$ & 68 & 2 \\
6 & $27^{\circ}$ & 36 & 1 \\
6 & $19^{\circ}$ & 17 & 1 \\
\hline
\end{tabular}

shapes. Concerning grain size, we adopt geometrically similar polyhedrons whose longer edges can be $1,1.5$ or $2 \mathrm{~mm}$ in length. Only one grain size is used in each granular flow.

Table 1 illustrates the combinations of the values of the channel width $w$, the lateral side inclination $\theta$, the grain size $\delta$ and the total granular flow mass adopted in the numerical simulations. In the channel with $w=6 \mathrm{~mm}$ and $\theta=27^{\circ}$, we use total granular flow masses equal to $8.9,13$ and $26 \mathrm{~g}$. This total mass increases in wider channels with $\theta=27^{\circ}$ : $29 \mathrm{~g}$ when $w=10 \mathrm{~mm}, 33$ and $68 \mathrm{~g}$ when $w=16 \mathrm{~mm}$, and 39 and $93 \mathrm{~g}$ when $w=26 \mathrm{~mm}$. The flow mobility of simulations with sidewall inclinations $\theta$ equal to 19 and $41^{\circ}$ (where $w=6 \mathrm{~mm}$ and $\delta=1 \mathrm{~mm}$ ) can be compared with the mobility obtained with $\theta=27^{\circ}$ and the same channel width $w$ and the same grain size $\delta$. In the simulations with $\theta$ equal to 19 and $41^{\circ}$, the total granular flow masses are 36 and $17 \mathrm{~g}$, respectively. Since particle density is a constant, the granular flow mass is proportional to flow volume, whose effect on mobility we are interested in. The actual granular mass values we use are not important in a scaling analysis, provided that the range of values is relatively wide so that the effect of volume on the collapse of the scaling parameters along a single
Table 2. Physical properties of materials.

\begin{tabular}{lrrr}
\hline & Particles & Channels* & Gates \\
\hline Poisson's ratio & 0.19 & 0.35 & 0.36 \\
Shear modulus (Pa) & $2.38 \times 10^{10}$ & $6.85 \times 10^{9}$ & $25 \times 10^{9}$ \\
Density $\left(\mathrm{kg} \mathrm{m}^{-3}\right)$ & 2700 & 2580 & 2700 \\
\hline
\end{tabular}

* Straight ramps and curved chutes have the same properties.

Table 3. Values of properties governing clast-clast, clast-channel and clast-gate interactions.

\begin{tabular}{lrrr}
\hline & Clast-clast & Clast-channel* $^{*}$ & Clast-gate \\
\hline $\begin{array}{l}\text { Coefficient of } \\
\text { restitution }\end{array}$ & 0.49 & 0.3 & 0.53 \\
$\begin{array}{l}\text { Coefficient of static } \\
\text { friction }\end{array}$ & 0.45 & 0.9 & 0.1 \\
$\begin{array}{l}\text { Coefficient of } \\
\text { rolling friction }\end{array}$ & 0.035 & 0.07 & 0.07 \\
$*$ Straight ramps and curved chutes have the same properties. &
\end{tabular}

* Straight ramps and curved chutes have the same properties.

line can be evaluated. Here we test a relatively large range of total granular mass values because the effect of volume on mobility is debated.

Table 2 shows the values of the physical properties of the materials we use in the numerical simulations, whereas Table 3 shows the values of the properties that govern particle-particle, particle-channel and particle-gate interactions. Here we are simulating flows of rock fragments that travel on a subsurface made of soil (Peng, 2000). The values shown in Tables 2 and 3 are the same as those adopted in the numerical simulations by Cagnoli and Piersanti (2015) because with these values it is possible to obtain the same relative mobility of the different flows as that observed in the laboratory by Cagnoli and Romano (2010, 2012a). The surface of the gates (made of aluminum) has a very small friction value to avoid disturbing the granular material when the gates are removed. The roughness of the chute and ramp surfaces is identical and everywhere it is significantly smaller than the smallest grain size we use.

We always adopt the same initial compaction and density of the granular material behind the gate before release by having the particles fill abstract spaces whose volumes are proportional to the granular masses. We have shown that the larger the initial compaction of the granular material behind the gate before release, the larger the mobility of the center of mass of the granular flow (Cagnoli and Piersanti, 2015). Therefore, it is important that the comparison of mobility is carried out among granular masses that before release have the same initial compaction. Here, behind the gate before release, the granular masses have a bulk average density equal to $721 \pm 14 \mathrm{~kg} \mathrm{~m}^{-3}$ and the particles are in random positions. The density of the particle material is $2700 \mathrm{~kg} \mathrm{~m}^{-3}$ 
(Table 2). Interestingly, the actual spatial arrangement of the randomly distributed particles behind the gate before release does not seem to make a difference because in the laboratory (Cagnoli and Romano, 2012a) different granular masses with the same features have the same mobility irrespective of this initial arrangement, which could not have been identi$\mathrm{cal}$. In the numerical simulations we use granular masses that are relatively small so that the computer processing times are manageable. The flows with the largest number of particles $(\sim 34000)$ are those with a total mass equal to $39 \mathrm{~g}$ and a grain size equal to $1 \mathrm{~mm}$. The flows with the smallest number of particles $(\sim 2800)$ are those with a total mass equal to $26 \mathrm{~g}$ and a grain size equal to $2 \mathrm{~mm}$.

\subsection{Three-dimensional discrete element software}

Our three-dimensional discrete element modeling was carried out by means of the software EDEM developed by DEM Solutions. EDEM uses the mass, volume and moment of inertia of the polyhedrons we chose, whereas it estimates impact forces during particle collisions as a function of the overlapping of sets of spheres that are inscribed within the polyhedrons (Fig. 4). These spheres are rigidly connected. Experience has shown that clusters of spheres are an effective method for modeling complex particle shapes with a good degree of approximation. These clusters of spheres provide a good approximation of angular fragments, probably because the clusters and the polyhedrons have a comparable distribution of their volumes in the different directions in space since the faces of the polyhedrons are tangent to the spheres (Fig. 4). Therefore, our numerical simulations pertain to angular fragments. It is well known that particle angularity affects particle friction (Lambe and Whitman, 1969; Abe and Mair, 2009). Interestingly, since both laboratory experiments (Cagnoli and Romano, 2012a) and numerical simulations (here and Cagnoli and Piersanti, 2015) generate the same grain size and flow volume effects, the angularity of the particles in the laboratory and in the numerical model must be equivalent. The EDEM contact model that governs particle-particle and particle-boundary interactions is based on the Hertzian contact theory and on the MindlinDeresiewicz studies (Hertz, 1882; Mindlin, 1949; Mindlin and Deresiewicz, 1953). The equations of this contact model are illustrated by DEM Solutions (2014) and in the paper by Cagnoli and Piersanti (2015).

\section{Scaling}

We measure the reciprocal of mobility of a flow by using the apparent coefficient of friction

$\mu_{\mathrm{A}}=\frac{h}{l}$,

where $h$ is the vertical drop of the center of mass of the granular material and $l$ is its horizontal distance of travel. Dis- tances $h$ and $l$ are measured from the position of the center of mass of the granular samples at rest behind the gate before release to the position of the center of mass of the final deposits (Fig. 1). In an energy dissipation (i.e., mobility) analysis, the knowledge of the whereabouts of the center of mass is a necessity because the center of mass is the only point that can be used as a proxy for the entire flow since it moves as though the total mass of all the particles were concentrated there and all the external forces were applied there. The positions in space of the centers of mass were computed here by means of a three-dimensional CAD software (Rhinoceros). Thus, our parameter differs from the so-called Heim coefficient that considers the vertical and horizontal distances between the highest elevation point of the failed landscape feature (a mountain slope or a volcanic dome, for example) and the most distal position of the front of the deposit (e.g., Scheidegger, 1973).

In both the laboratory experiments (Cagnoli and Romano, 2012a) and the numerical simulations (here and in Cagnoli and Piersanti, 2015), the deposited granular material consists of the deposit of the flow proper and a more distal distribution of individual fragments. The value of $\mu_{\mathrm{A}}$ is computed for the final deposit of the flow proper, which consists of all the particles on the chute that are in contact with one another. However, for comparison, we also show the value of $\mu_{\mathrm{A}}$ computed for the combined particles of the final deposit of the flow proper and the distal distribution together.

We are interested here in a functional relation between the apparent coefficient of friction $\mu_{\mathrm{A}}$, the grain size $\delta$, the flow volume $V$, the channel width $w$ and the length of the flow $L$ :

$f\left(\mu_{\mathrm{A}}, \delta, V, w, L\right)=0$.

The length of the flow $L$ is considered to be the pertinent length scale for the flow as a whole (Iverson et al., 2010). We introduce $L$ here to include in the analysis the extent of the entire spread of the flow particles along the longitudinal direction of the channel. The width $w$ gives the same information along the transversal direction. The combined presence of $L$ and $w$ is instrumental in providing information on the number density of the particles within the flows. In general, granular flow mobility is affected by many more quantities whose values are, however, held deliberately constant in our system as discussed in Sect. 1 and in Sect. 5.4.

Equation (3) has five quantities with one fundamental physical dimension (length). For this reason, according to the Buckingham Pi theorem (e.g., Dym, 2004), it is equivalent to a functional relation containing four dimensionless parameters. Since the grain size $\delta$ is a key quantity ( $\delta$ is the pertinent length scale for the grain-scale mechanics that generate the stresses; Iverson et al., 2010), we use it to scale the other variables so that Eq. (3) is replaced by the following functional relation $F$ :

$\mu_{\mathrm{A}}=F\left(\frac{V^{1 / 3}}{\delta}, \frac{L}{\delta}, \frac{w}{\delta}\right)$. 


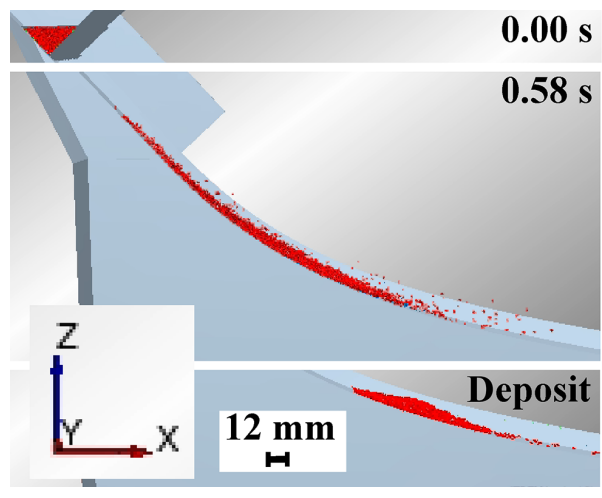

Figure 5. Cross sections illustrating three moments of a flow history. The frame at the top shows the granular material at rest behind the gate before release. The frame in the middle shows the fully developed shape of travel of the granular flow. The lower frame shows the final deposit. In this example the granular mass is $93 \mathrm{~g}$, the grain size is $2 \mathrm{~mm}, w$ is $26 \mathrm{~mm}$ and $\theta$ is $27^{\circ}$. The values measured in seconds show the time elapsed since the gate removal.

Our numerical simulations show that the use of these dimensionless parameters results in a successful collapse of the data along a single fitting straight line:

$\mu_{\mathrm{A}}=a \chi+b$,

where

$\chi=\frac{V^{1 / 3} \delta}{L w}$

and $a$ and $b$ are coefficients. Parameter $\chi$ is a ratio of the three independent dimensionless parameters of Eq. (4) and it can be expressed as the ratio of quantities $\Gamma$ and $\gamma$ :

$\chi=\frac{V^{1 / 3} \delta}{L w}=\frac{V^{1 / 3}}{\delta} \frac{\delta}{L} \frac{\delta}{w}=\frac{V^{1 / 3}}{\delta} \frac{\delta^{2}}{L w}=\frac{\Gamma}{\gamma}$,

where

$\Gamma=\frac{V^{1 / 3}}{\delta}$

and

$\gamma=\frac{L w}{\delta^{2}}$

The dimensionless quantity $\Gamma$ is an increasing function of the total number of particles in the flow because it corresponds to $V / \delta^{3}$. The dimensionless quantity $\gamma$ is an increasing function of the number of particles per unit of flow volume (i.e., flow mass). This is because $\gamma$ is proportional to the number of particles whose sum of cross-sectional area completely covers a characteristic surface area of the flow equal to $L w$ (the central cross-sectional area of a particle is proportional to $\delta^{2}$ ). Here, quantity $V$ is obtained by summing the individual volumes of all the polyhedrons that form the deposit of the flow proper so that, since the particle density is a constant, this $V$ value is proportional to the flow mass.

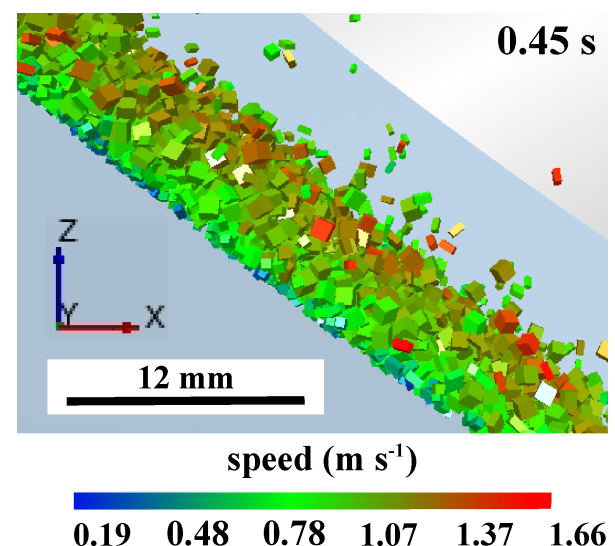

Figure 6. Side view of a granular flow. Particle speed decreases toward the subsurface along its normal. In this example the granular mass is $26 \mathrm{~g}$, the grain size is $1 \mathrm{~mm}, w$ is $6 \mathrm{~mm}$ and $\theta$ is $27^{\circ}$. The time measured in seconds is that elapsed since the gate removal.

\section{Results}

The flows can be examined in cross section (Figs. 5 and 6) or from the top (Figs. 7 and 8) because the numerical simulations are three-dimensional. As soon as the gate is removed, the granular mass accelerates down the slope, reaches a maximum speed and then decelerates and stops (Fig. 5). Figure 6 shows that the slope-parallel component of particle velocity within the flows decreases toward the subsurface along its normal, as expected in flows that travel in contact with a boundary surface (Iverson, 2003; Cagnoli and Romano, 2013). Particles that are not part of the flow proper because they travel individually and form, when at rest, the distal distribution are clearly discernible in the numerical simulations at the front and at the back of the flow proper (Figs. 7 and 8).

The reciprocal of mobility $\mu_{\mathrm{A}}$ is plotted versus parameter $\chi$ in Figs. 9 and 10. The values of $\mu_{\mathrm{A}}$ in these figures are relatively large because they refer to the final position of the center of mass and not to the final position of the front of the deposit (the flow front is more mobile than the center of mass). We have also adopted a relatively large basal friction value (Table 3). The successful collapse along a single straight line of all the data points of the simulations with $\theta=27^{\circ}$ confirms that, in Figs. 9 and 10, only the variables considered in Eq. (6) have values that vary and consequently determine the observed different mobility of the center of mass of the different flows.

In the figures of this paper, we have added to each $\mu_{\mathrm{A}}$ value of the flow proper an uncertainty bar whose extremities are computed as follows. The upper extremity is the $h / L$ value of the portion of deposit whose distal end is $3 \mathrm{~cm}$ in a more proximal position than the distal end of our best estimate of the deposit of the flow proper. The lower extremity is the $h / l$ value of all the particles on the chute (deposit of the flow proper plus distal distribution). The $3 \mathrm{~cm}$ shorter deposit 


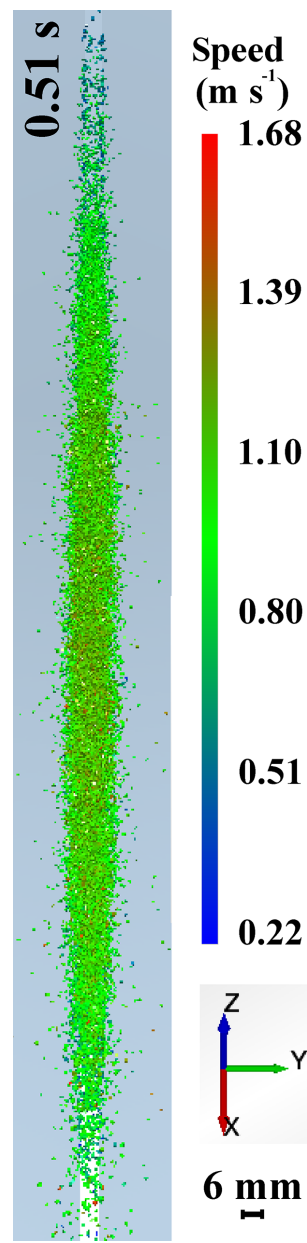

Figure 7. Fully developed shape of travel of a granular flow seen from above $0.51 \mathrm{~s}$ after the gate removal. In this example, the granular mass is $26 \mathrm{~g}$, the grain size is $1 \mathrm{~mm}, w$ is $6 \mathrm{~mm}$ and $\theta$ is $27^{\circ}$.

and all the particles on the chute provide $h / l$ values that can be significantly different from the true $\mu_{\mathrm{A}}$ value of the flow proper. Therefore, the true $\mu_{\mathrm{A}}$ value of the flow proper is located near our best estimate.

In Fig. 9, parameter $\chi$ is computed by setting $L$ equal to the length of the deposits. In this figure, Eq. (5) has a coefficient $a$ equal to 0.95 and a coefficient $b$ equal to 0.81 . The linear correlation coefficient $r$ (Taylor, 1997) of the fitting straight line in this figure is equal to 0.93 . Since the probability that 16 values for each of two uncorrelated variables generate an $r$ value equal to 0.93 is much less than $0.05 \%$ (Taylor, 1997), the linear correlation between $\mu_{\mathrm{A}}$ and $\chi$ in Fig. 9 is highly significant.

In Fig. 10, parameter $\chi$ is computed by setting $L$ equal to the length of the flows (measured $0.58 \mathrm{~s}$ after the gate removal in all simulations). In this figure, Eq. (5) has a coefficient $a$ equal to 2.05 and a coefficient $b$ equal to 0.81 . The linear correlation coefficient $r$ (Taylor, 1997) of this fitting straight line is equal to 0.91 . Since the probability that 16

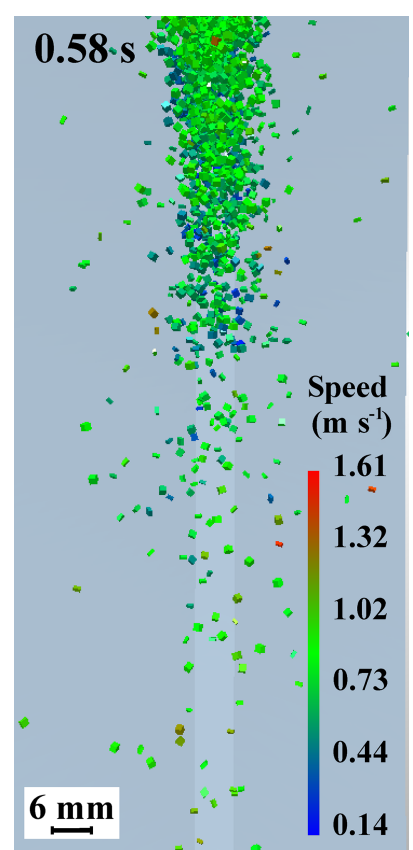

Figure 8. Flow front seen from above $0.58 \mathrm{~s}$ after the gate removal. The saltating particles of the distal distribution in front of the dense granular flow are clearly identifiable. In this example the granular mass is $26 \mathrm{~g}$, the grain size is $1 \mathrm{~mm}, w$ is $6 \mathrm{~mm}$ and $\theta$ is $27^{\circ}$.

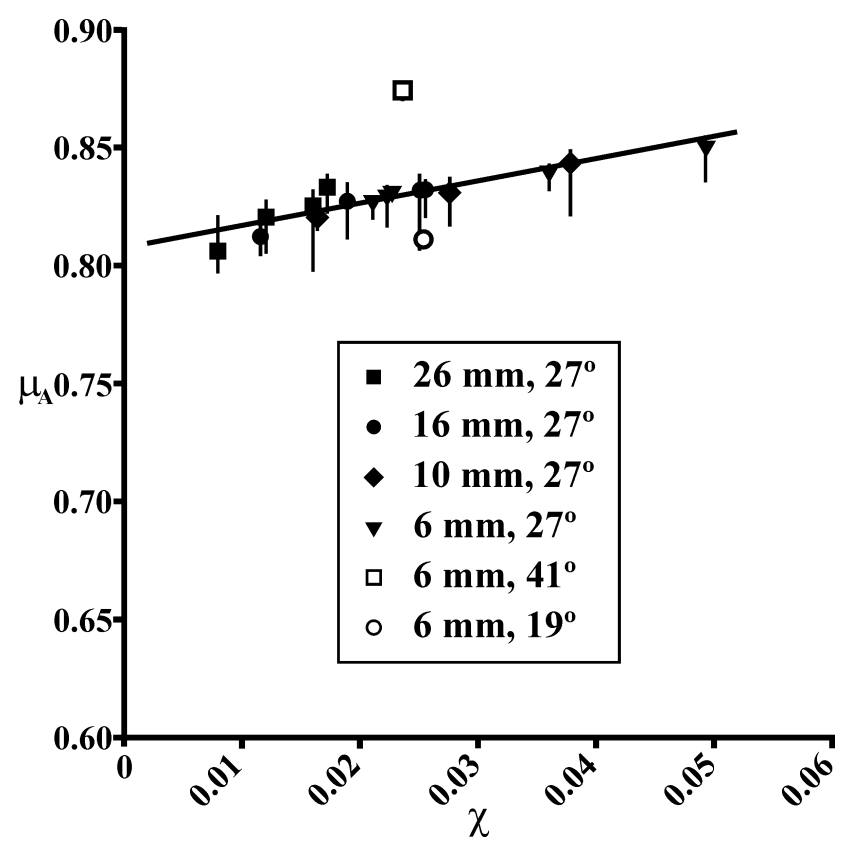

Figure 9. Plot of the reciprocal of mobility $\mu_{\mathrm{A}}$ versus parameter $\chi$, where $L$ is set equal to the length of the deposit. The values in millimeters are the channel widths $w$ and the values in degrees are the sidewall inclinations $\theta$. When the uncertainty bar is not visible, it is smaller than the symbol used. The straight line only fits (by means of the least squares method) the results of the simulations with $\theta=27^{\circ}$. 


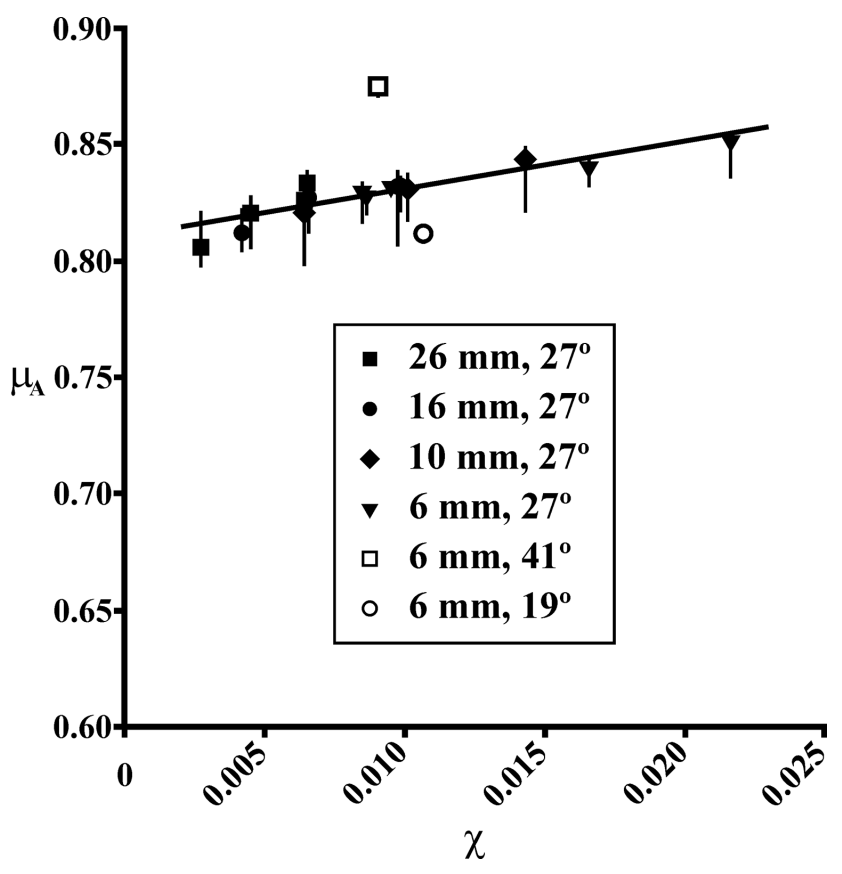

Figure 10. Plot of the reciprocal of mobility $\mu_{\mathrm{A}}$ versus parameter $\chi$, where $L$ is set equal to the length of the flows. This length was measured $0.58 \mathrm{~s}$ after the gate removal in all flows (i.e., when they already had a fully developed shape of travel). Figures 9 and 10 illustrate the same numerical simulations. The values in millimeters are the channel widths $w$ and the values in degrees are the sidewall inclinations $\theta$. When the uncertainty bar is not visible, it is smaller than the symbol used. The straight line only fits (by means of the least squares method) the results of the simulations with $\theta=27^{\circ}$.

values for each of two uncorrelated variables generate an $r$ value equal to 0.91 is less than $0.05 \%$ (Taylor, 1997), the linear correlation between $\mu_{\mathrm{A}}$ and $\chi$ in Fig. 10 is also highly significant.

In Figs. 9 and 10, the straight line only fits the simulation results in channels with different width $w$ but the same sidewall inclination $\theta$ (in this case equal to $27^{\circ}$ ). The results of simulations in channels with different sidewall inclinations (here 19 and $41^{\circ}$ ) plot along different straight lines (we expect one straight line for each sidewall inclination) since parameter $\chi$ does not consider $\theta$ because $\theta$ is itself another distinct and independent dimensionless scaling parameter. Figures 9 and 10 show that the larger the value of the sidewall inclination $\theta$, the larger the value of $\mu_{\mathrm{A}}$.

\section{Discussion}

\subsection{Grain size effect on flow mobility}

The results of the numerical simulations illustrated in this paper (Figs. 9 and 10) confirm that the finer the grain size (all the other features being the same), the more mobile the center of mass of the granular flows (Cagnoli and Romano, 2010,

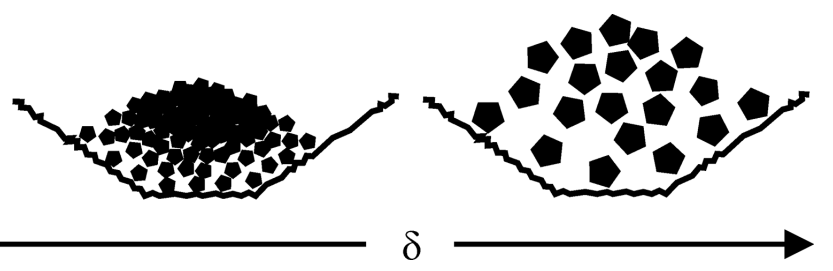

Figure 11. These illustrations portray particle agitation per unit of flow mass that increases as grain size $\delta$ increases (all the other features being the same). In this figure, the more agitated particles are represented by more distant polygons. The closer the position to the rough channel surfaces, the larger the agitation of the particles. These sketches are based on high-speed video camera studies of granular flows (Cagnoli and Romano, 2012a, b).

2012a). This is due to the fact that the finer the grain size, the less agitated the particles per unit of flow mass so that finer grain size flows dissipate less energy per unit of travel distance (Cagnoli and Romano, 2010). Particles are less agitated because in finer grain size flows (all the other features being equal, including the total flow mass) there is a larger number of clasts per unit of flow mass, and for this reason the agitation due to the interaction with the rough subsurface and the rough sidewalls penetrates relatively less inside the flows (Fig. 11). It is for this reason that the reciprocal of mobility $\mu_{\mathrm{A}}$ is inversely proportional to $\gamma$ (Eq. 5), where $\gamma$ is an increasing function of the number of particles per unit of flow mass.

In the laboratory, the increase in particle agitation as grain size increases (all the other features being the same) is visually noticeable in the high-speed video camera images of the granular flows and it was assessed quantitatively by (1) measuring particle agitation as the normalized average squared deviation from the mean of the particle transversal speeds and (2) measuring the fluctuations of granular pressure at the base of the flows (Cagnoli and Romano, 2010, 2012b). Speed and energy calculations in numerical simulations also confirm that flows with coarser fragments have more agitated particles and are energetically more dissipative than flows of finer particles, with all the other features being the same (Cagnoli and Piersanti, 2015). Particle agitation affects energy dissipation through particle-particle and particle-boundary interactions (friction and collisions) and through diversion of energy into directions (the transversal one for example) that are different from the downslope direction.

Numerical simulations (Mollon et al., 2015) show that also on laterally unconfined planar slopes ending on a depositional horizontal plane with no lateral constraints, an increase in particle size tends to decrease the mobility of the center of mass when a collisional regime is allowed to occur. In debris flows, the presence of intergranular clay and water can alter this trend because of the activation of a different rheology as the fraction of the coarse grains decreases. De Haas 
et al. (2015) experimentally found that in debris flows, as the content of coarse grains (gravel in their case) decreases, the residual slurry is not able to build lateral levees. This means that below a critical gravel content, the lateral spreading on unconfined slopes increases and consequently the longitudinal runout decreases.

In our paper, we investigate the effect of grain size on flow mobility. This has been carried out by holding constant the value of grain size during flow motion to prevent other phenomena from interfering with the grain size effect under study. However, we believe that in nature particle-particle and particle-boundary interactions cause particle fragmentation during flow motion. We observed this phenomenon in granular flows during laboratory experiments (see Fig. 7 in Cagnoli and Manga, 2004). In rock avalanches, there is field evidence that grain size decreases as travel distance increases and that flows with long runouts are associated with a reduced grain size (Davies and McSaveney, 2009; Zhang et al., 2016). In particular, Davies and McSaveney (2009) advance a theory in which they suggest that the fragmentation process in itself can increase flow mobility. However, our research (by means of both laboratory experiments and numerical simulations) demonstrates that even if rock fragmentation does not occur, the mere presence of fine grain size is conducive to a reduced energy dissipation of the granular flows. Interestingly, this grain size effect is also observed when the grain size in the flows has a relatively narrow distribution of its values, such as in the laboratory experiments by Cagnoli and Romano (2012a) where the following grain size ranges were used: $0.5-1,1-2$ and 2-3 mm.

\subsection{Flow volume effect on flow mobility}

Figures 9 and 10 also confirm that there is a decrease in mobility of the center of mass as flow volume increases with all the other features being the same (Cagnoli and Romano, 2012a; Cagnoli and Piersanti, 2015). This effect is the result of the fact that a deposit accretes backward during its formation on a change of slope. In this case, the larger the quantity of granular material that accumulates at the back (i.e., the larger the flow volume), the longer the backward shift of the center of mass (Cagnoli and Romano, 2012a). It is for this reason that the reciprocal of mobility $\mu_{\mathrm{A}}$ is directly proportional to $\Gamma$ (Eq. 5), where $\Gamma$ is an increasing function of the total number of particles in the flow. The backward accretion is due to the fact that the front of the flow reaches the lesssteep part of the curved slope and stops before the rear part, preventing the rear part and the center of mass from traveling further downhill, as revealed by high-speed video camera images in the laboratory experiments and as clearly visible in the numerical simulations.

The volume effect we observe occurs when deposition takes place wherever there is a change in the slope inclination angle, either gradual (here and in Cagnoli and Romano, 2012a) or abrupt (Okura et al., 2000). Okura et al. (2000) ob- tained this effect on a laterally unconfined planar slope ending on a horizontal depositional plane with no lateral constraints. With such sharp breaks in slope, the larger the decrease in the slope inclination angle, the larger the energy dissipation of the flow (Manzella and Labiouse, 2013). In nature, the flank of a volcano provides an instance of a gradual change in the slope inclination angle (besides the flanks of Mayon Volcano, other examples illustrated in the literature are those of Mount Ngauruhoe (Lube et al., 2007) and Volcán de Colima (Saucedo et al., 2002)), whereas the intersection of a mountainside with an alluvial plain is an example of an abrupt change in the slope inclination angle. When assessing the volume effect in laboratory experiments and numerical simulations, the measure of mobility of the flows must take into account that within the same container at the top of the slope before release, larger volumes have an unavoidably higher elevation of their center of mass for a geometric reason. In parameter $\mu_{\mathrm{A}}$ (Eq. 2), flow mobility is indeed normalized by the different initial elevations $h$ of the center of mass of the different granular volumes.

\subsection{Channel width effect on flow mobility}

In our previous publications (Cagnoli and Romano, 2012a; Cagnoli and Piersanti, 2015), we introduced a scaling parameter $\beta=\delta V^{1 / 3} / w^{2}$, which has the same numerator of $\chi$ but a different denominator. These previous publications focused exclusively on the effects of grain size $\delta$ and flow volume $V$ (i.e., the numerator of $\beta$ ). The denominator of $\beta$ was conjectural since the channel width $w$ did not vary. This conjecture was however based on the observation that with a change in slope, the front of a granular flow stops before the rear. This means that the deposit propagates backward and, the narrower the channel, the longer the deposit and, thus, the longer the backward migration of the centers of mass during deposition. In other words, although the channel width is expected to be at the denominator because the smaller its value, the smaller the mobility of the center of mass, a specific set of investigations where the value of $w$ varies systematically is necessary to figure out its exponent. This paper illustrates this set of investigations.

The plot of $\mu_{\mathrm{A}}$ versus $\beta$ shows that the exponent of quantity $w$ is different from 2. In Fig. 12, simulations with the same channel width plot along the same straight line (thus confirming the same grain size and flow volume effects of Figs. 9 and 10), but the straight lines for different channel widths have different gradients. Therefore, in parameter $\beta$, an exponent of $w$ equal to 2 falls short of satisfactorily accommodating the channel width effect.

The simulations carried out with different channel widths show that the exponent of $w$ is equal to 1 so that another quantity has to be introduced at the denominator of $\chi$ for this parameter to be dimensionless. Here, this quantity $L$ is either the length of the deposits (Fig. 9) or the length of the flows (Fig. 10). Both length values can be used because when 


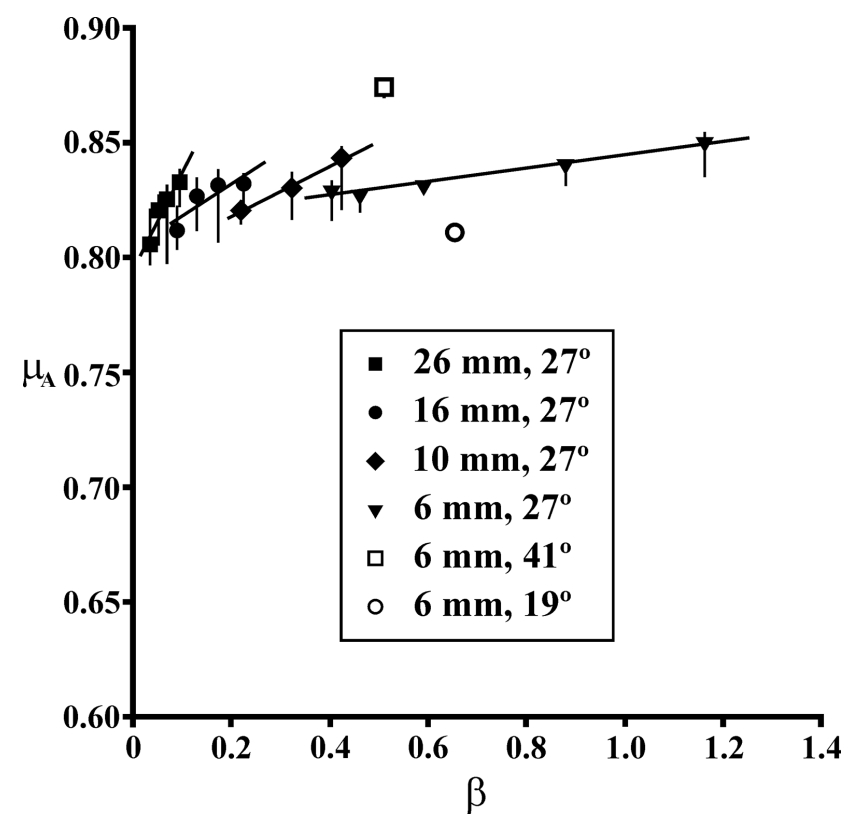

Figure 12. Plot of the reciprocal of mobility $\mu_{\mathrm{A}}$ versus parameter $\beta$ of the same flow simulations illustrated in Figs. 9 and 10. The values in millimeters are the channel widths $w$ and the values in degrees are the sidewall inclinations $\theta$. When the uncertainty bar is not visible, it is smaller than the symbol used. Each straight line fits (by means of the least squares method) the results of simulations with the same channel width $w$, but only those with $\theta=27^{\circ}$.

comparing model and nature, one is expected to scale as the other does. The length of the flow is however difficult to assess in the field (where pyroclastic flows and rock avalanches are hidden by clouds of fine particles and they are too dangerous to approach at close range) and in the laboratory or in the numerical simulations (where there are numerous isolated particles at the front and at the back of the flows so that it is difficult to decide where the transition between the different transport mechanisms of saltating particles and the dense flow proper occurs). It is for this reason that in Fig. 10, where $\chi$ is computed by using the length of the flows, the linear correlation coefficient $r$ is smaller than that in Fig. 9, where $\chi$ is computed by using the length of the deposits.

It is important to realize that parameters $\chi$ and $\beta$ have the same meaning because they both imply that the mobility of the center of mass of the granular flows is an increasing function of the ratio of the number of particles per unit of flow mass to the total number of fragments in the flow (Cagnoli and Romano, 2012a). Notably, the characteristic surface area $L w$ in $\chi$ is actually more appropriate than $w^{2}$ in $\beta$ to uphold that

$\mu_{\mathrm{A}} \propto \frac{\Gamma}{\gamma}$

because $L w$ considers the complete spread of all the particles along the entire flow length. Quantity $L$ cannot be directly set as an initial condition by the experimenter in the laboratory or the modeler in the numerical simulations because it is determined by the other channel and flow features that govern the spread of the particles within the channel (including the channel width, the grain size and the flow volume). Quantity $L$ has been, however, introduced in Eq. (3) because the knowledge of $V$ and $w$ alone does not suffice to reckon the extent of the longitudinal spread of the particles within the channel since the thickness of the flow is missing in this equation.

In our simulations, all our flows are channeled and we compare their mobility in channels with different widths. The narrower the channel, the less mobile the center of mass. This is because the deposit propagates backward during its formation and the narrower the channel, the longer the backward propagation. It is also possible that in a narrower channel the retarding effect of the sidewalls is relatively more important. However, a different mechanism explains the different behaviors that are observed when comparing the mobility of channeled flows and the mobility of flows without lateral constraints. In this case, the flows without lateral constraints tend to be less mobile (Nicoletti and Sorriso-Valvo, 1991). This is due to the fact that a flow without lateral constraints spreads laterally, whereas the entire momentum of a channeled flow is spent along a single direction: that of the channel.

\subsection{Other quantities affecting flow mobility}

In general, besides grain size, flow volume and channel width, the mobility of the center of mass of a granular flow is a function of several other variables as well. These variables include initial flow speed $s$ (which is equal to zero in all our flows), acceleration of gravity $g$, density $\rho_{\mathrm{S}}$ of the particles, density $\rho_{\mathrm{f}}$ of the intergranular fluid, dynamic viscosity $\eta$ of the intergranular fluid, angle of internal friction $\varphi$ of the particles, coefficient of restitution $e$ of the rock material, average height $i$ (the roughness) of the subsurface and sidewall asperities, shape and surface texture of the particles, and intergranular fluid pressure. However, in our calculations these variables have either a constant value (such as $s, g, \rho_{\mathrm{s}}, \varphi, e$, $i$ and the shape and the surface texture of the grains) or they refer to an intergranular fluid ( $\rho_{\mathrm{f}}, \eta$ and its pressure) that is absent in the numerical simulations either as a liquid or as a gas.

Concerning the intergranular fluid, the numerical simulations described in this paper demonstrate that the grain size effect and the flow volume effect we observe in the laboratory (Cagnoli and Romano, 2012a) are not due to the presence of either an intergranular gas or an intergranular liquid. This is because the numerical simulations where an intergranular fluid is absent (here and in Cagnoli and Piersanti, 2015) do nonetheless predict the same relative flow mobility observed in the laboratory where an intergranular fluid (air) is present. Therefore, it is important to note that finer grain size 
flows dissipate intrinsically less energy than coarser grain size flows, irrespective of the presence of an intergranular fluid (either gaseous or liquid).

Figures 9 and 10 show that the sidewall inclination $\theta$ also affects granular flow mobility. However, the angle $\theta$ does not enter parameter $\chi$ because $\theta$ is a distinct and independent scaling parameter. We thus expect a different $\chi$ vs. $\mu_{\mathrm{A}}$ linear relation for each different $\theta$ value. Figures 9 and 10 indicate that the larger $\theta$, the smaller the mobility of the center of mass.

\section{Conclusions}

Our new three-dimensional numerical simulations of granular flows of angular rock fragments confirm that (all the other features being equal) (1) the finer the grain size, the larger the mobility of the flow center of mass; (2) the smaller the flow volume, the larger the mobility of the flow center of mass and (3) the wider the channel, the larger the mobility of the flow center of mass. The proportionality between the reciprocal of mobility $\mu_{\mathrm{A}}$ and scaling parameter $\chi$ quantitatively expresses these three effects on granular flow mobility. Here we show that the same type of grain size effect and the same type of flow volume effect occur regardless of the value of the channel width. The main new contribution of this paper consists of showing that the channel width occurs at the denominator of this scaling parameter with an exponent equal to 1 . The other quantity that enters the denominator of this parameter to make it dimensionless is the length of the deposit or the length of the flow.

Our model granular flows are relevant for natural systems where the flows acquire a fully developed travel shape after the initial deformation of the failed landscape feature (such as a mountainside or a volcanic dome) and before the final deposition. This fully developed shape of travel of a flow proper consists of a finite contiguous body of rock fragments traveling in unison. The grain size, the flow volume and the channel width effects illustrated here are scale invariant because they are a function of only characteristic numbers of particles that since they are dimensionless, determine the distinctive character of any flow at any scale, as also suggested by the generalization provided by the numerical simulations. In particular, the mobility of the center of mass of granular flows is confirmed to be an increasing function of the ratio of the number of particles per unit of flow mass to the total number of particles in the flow.

\section{Data availability}

The data upon which the conclusions of this paper are based are available from the corresponding author on request.
Competing interests. The authors declare that they have no conflict of interest.

Acknowledgements. We thank Tjalling de Haas, Ming Zhang and Steffen Abe for their useful comments.

Edited by: T. Gerya

Reviewed by: M. Zhang and S. Abe

\section{References}

Abe, S. and Mair, K.: Effects of gouge fragment shape on fault friction: New 3D modelling results, Geophys. Res. Lett., 36, L23302, doi:10.1029/2009GL040684, 2009.

Banton, J., Villard, P., Jongmans, D., and Scavia, C.: Twodimensional discrete element models of debris avalanches: Parameterization and the reproducibility of experimental results, J. Geophys. Res., 114, F04013, doi:10.1029/2008JF001161, 2009.

Becker, G. F.: A feature of Mayon Volcano, Proc. Wash. Acad. Sci., 7, 277-282, 1905.

Cagnoli, B. and Manga, M.: Granular mass flows and Coulomb's friction in shear cell experiments: Implications for geophysical flows, J. Geophys. Res., 109, F04005, doi:10.1029/2004JF000177, 2004.

Cagnoli, B. and Piersanti, A.: Grain size and flow volume effects on granular flow mobility in numerical simulations: 3-D discrete element modeling of flows of angular rock fragments, J. Geophys. Res. Solid Earth, 120, 2350-2366, doi:10.1002/2014JB011729, 2015.

Cagnoli, B. and Romano, G. P.: Effect of grain size on mobility of dry granular flows of angular rock fragments: An experimental determination, J. Volcanol. Geotherm. Res., 193, 18-24, 2010.

Cagnoli, B. and Romano, G. P.: Effects of flow volume and grain size on mobility of dry granular flows of angular rock fragments: A functional relationship of scaling parameters, J. Geophys. Res., 117, B02207, doi:10.1029/2011JB008926, 2012a.

Cagnoli, B. and Romano, G. P.: Granular pressure at the base of dry flows of angular rock fragments as a function of grain size and flow volume: A relationship from laboratory experiments, J. Geophys. Res., 117, B10202, doi:10.1029/2012JB009374, 2012b.

Cagnoli, B. and Romano, G.P.: Vertical segregations in flows of angular rock fragments: Experimental simulations of the agitation gradient within dense geophysical flows, J. Volcanol. Geotherm. Res., 265, 52-59, 2013.

Cas, R. A. F. and Wright, J. V.: Volcanic Successions, Unwin Hyman, London, 1988.

D'Agostino, V., Cesca, M., and Marchi, L.: Field and laboratory investigations of runout distances of debris flows in the Dolomites (Eastern Italian Alps), Geomorphology, 115, 294-304, 2010.

Davies, T. R.: Spreading of rock avalanche debris by mechanical fluidization, Rock Mech., 15, 9-24, 1982.

Davies, T. R. and McSaveney, M. J.: The role of rock fragmentation in the motion of large landslides, Eng. Geol., 109, 67-79, 2009.

De Haas, T., Braat, L., Leuven, J. R. F. W., Lokhorst, I. R., and Kleinhans, M. G.: Effects of debris flow composition on runout, 
depositional mechanisms, and deposit morphology in laboratory experiments, J. Geophys. Res. Earth Surf., 120, 1949-1972, doi:10.1002/2015JF003525, 2015.

DEM Solutions: EDEM 2.6 Theory Reference Guide, Edinburgh, United Kingdom, 2014.

Dym, C. L.: Principles of Mathematical Modeling, Elsevier Academic Press, San Diego, California, 2004.

Girolami, L., Hergault, V., Vinay, G., and Wachs A.: A threedimensional discrete-grain model for the simulation of dambreak rectangular collapses: Comparison between numerical results and experiments, Granul. Matter, 14, 381-392, 2012.

Griswold, J. P. and Iverson, R. M.: Mobility statistics and automated hazard mapping for debris flows and rock avalanches, U.S. Geological Survey Scientific Investigations, Report 2007-5276, 2008.

Hall, M. L., Steele, A. L., Bernard, B., Mothes, P. A., Vallejo, S. X., Douillet, G. A., Ramón, P. A., Aguaiza, S. X., and Ruiz, M. C.: Sequential plug formation, disintegration by Vulcanian explosions, and the generation of granular Pyroclastic Density Currents at Tungurahua volcano (2013-2014), Ecuador, J. Volcanol. Geotherm. Res., 306, 90-103, 2015.

Hertz, H.: Ueber die Berührung fester elastischer Körper, J. Reine Angew. Math., 92, 156-171, 1882.

Hungr, O., Leroueil, S., and Picarelli, L.: The Varnes classification of landslide types, an update, Landslides, 11, 167-194, 2014.

Iverson, R. M.: Gravity-driven mass flows, in: Encyclopedia of Sediments and Sedimentary Rocks, edited by: Middleton, G. V., 347-353, Kluwer Academic Publishers, Dordrecht, 2003.

Iverson, R. M., Logan, M., LaHusen, R. G., and Berti, M.: The perfect debris flow? Aggregated results from 28 large-scale experiments, J. Geophys. Res., 115, F03005, doi:10.1029/2009JF001514, 2010.

Lambe, T. W. and Whitman, R. V.: Soil Mechanics, John Wiley and Sons, New York, 1969.

Lube, G., Cronin, S. J., Platz, T., Freundt, A., Procter, J. N., Henderson, C., and Sheridan, M. F.: Flow and deposition of pyroclastic granular flows: A type example from the 1975 Ngauruhoe eruption, New Zealand, J. Volcanol. Geotherm. Res., 161, 165-186, 2007.

Manzella, I. and Labiouse, V.: Empirical and analytical analyses of laboratory granular flows to investigate rock avalanche propagation, Landslides, 10, 23-36, 2013.

Mead, S. R. and Cleary, P. W.: Validation of DEM prediction for granular avalanches on irregular terrain, J. Geophys. Res. Earth Surf., 120, 1724-1742, doi:10.1002/2014JF003331, 2015.
Mindlin, R. D.: Compliance of elastic bodies in contact, J. Appl. Mech., 16, 259-268, 1949.

Mindlin, R. D. and Deresiewicz, H.: Elastic spheres in contact under varying oblique forces, J. Appl. Mech., 20, 327-344, 1953.

Mollon, G., Richefeu, V., Villard, P., and Daudon, D.: Numerical simulation of rock avalanches: Influence of a local dissipative contact model on the collective behavior of granular flows, J. Geophys. Res., 117, F02036, doi:10.1029/2011JF002202, 2012.

Mollon, G., Richefeu, V., Villard, P., and Daudon, D.: Discrete modelling of rock avalanches: sensitivity to block and slope geometries, Granul. Matter, 17, 645-666, 2015.

Nairn, I. A. and Self, S.: Explosive eruptions and pyroclastic avalanches from Ngauruhoe in February 1975, J. Volcanol. Geotherm. Res., 3, 39-60, 1978.

Nicoletti, P. G. and Sorriso-Valvo, M.: Geomorphic controls of the shape and mobility of rock avalanches, Geol. Soc. Am. Bull., 103, 1365-1373, 1991.

Okura, Y., Kitahara, H., Sammori, T., and Kawanami, A.: The effects of rockfall volume on runout distance, Eng. Geol., 58, 109124, 2000.

Peng, B.: Rockfall trajectory analysis: Parameter determination and application, M.S. thesis, University of Canterbury, Christchurch, New Zealand, 2000.

Saucedo, R., Macías, J. L., Bursik, M. I., Mora, J. C., Gavilanes, J. C., and Cortes, A.: Emplacement of pyroclastic flows during the 1998-1999 eruption of Volcán de Colima, México, J. Volcanol. Geotherm. Res., 117, 129-153, 2002.

Scheidegger, A. E.: On the prediction of the reach and velocity of catastrophic landslides, Rock Mech., 5, 231-236, 1973.

Taylor, J. R.: An Introduction to Error Analysis, University Science Books, Sausalito, California, 1997.

Valentino, R., Barla, G., and Montrasio, L.: Experimental analysis and micromechanical modelling of dry granular flow and impacts in laboratory flume tests, Rock Mech. Rock Eng., 41, 153-177, 2008.

Yohannes, B., Hsu, L., Dietrich, W. E., and Hill, K. M.: Boundary stresses due to impacts from dry granular flows, J. Geophys. Res., 117, F02027, doi:10.1029/2011JF002150, 2012.

Zhang, M. and Yin, Y.: Dynamics, mobility-controlling factors and transport mechanisms of rapid long-runout rock avalanches in China, Eng. Geol., 167, 37-58, 2013.

Zhang, M., Yin, Y., and McSaveney, M.: Dynamics of the 2008 earthquake-triggered Wenjiagou Creek rock avalanche, Qingping, Sichuan, China, Eng. Geol., 200, 75-87, 2016. 